\title{
Electrochemical Characteristics of Micrometer-sized Sn and Acetylene Black Composites Prepared by Mechanical Milling for Sodium-ion Battery Anodes
}

\section{Ryoji MARUBAYASHI, ${ }^{a, *, \S}$ Yoshitsugu YAMAMOTO, ${ }^{a}$ and Mitsuhiro HIROTA ${ }^{b}$}

${ }^{a}$ Energy Technology Section, Mie Prefecture Industrial Research Institute, 5-5-45 Takajaya, Tsu, Mie 514-0819, Japan

b Engineering Development Department, Tris Incorporated, 1105-2 Kushida-cho, Matsusaka, Mie 515-0204, Japan

* Corresponding author: marubr00@pref.mie.Ig.jp

\begin{abstract}
Five types of micrometer-sized $\mathrm{Sn}$ and acetylene black (AB) composite powders were prepared by mechanical milling for $1,3,6,12$, and $24 \mathrm{~h}$. The Sn/AB powders obtained, in addition to Sn-only powders were added to a binder and conductive material, and then dried under vacuum to prepare negative electrodes (anodes) for sodium-ion batteries (SIBs). SIBs were fabricated with the anodes in the form of 2032type coin cells, and were evaluated using charge-discharge tests up to 50 cycles within the cutoff voltage range of $0.005-0.65 \mathrm{~V}$ at a constant current of $50 \mathrm{~mA} \mathrm{~g}^{-1}$ at $25^{\circ} \mathrm{C}$. Maximum discharge capacities of 614 to $651 \mathrm{mAh} \mathrm{g}^{-1}$ were obtained with all the anodes prepared with both the Sn-only and the Sn/AB composites. However, the discharge capacities of the Sn-only and Sn/AB composites milled for 1 and $3 \mathrm{~h}$ were significantly decreased as the charge-discharge cycle increased. In contrast, the Sn/AB composites milled for $6 \mathrm{~h}$ or more exhibited improved cycle characteristics; capacities of 635,619 , and $584 \mathrm{mAh} \mathrm{g}^{-1}$ were maintained during 50 cycles of testing with the Sn/AB_6h, $\mathrm{Sn} / \mathrm{AB} \_12 \mathrm{~h}$, and $\mathrm{Sn} / \mathrm{AB} \_24 \mathrm{~h}$ samples, respectively, which were significantly higher than the anode prepared with the Sn-only powder $\left(135 \mathrm{mAh} \mathrm{g}^{-1}\right)$.
\end{abstract}

(C) The Author(s) 2021. Published by ECSJ. This is an open access article distributed under the terms of the Creative Commons Attribution 4.0 License (CC BY, $\mathrm{http}: / /$ creativecommons.org/licenses/by/4.0/), which permits unrestricted reuse of the work in any medium provided the original work is properly cited. [DOI: 10.5796/electrochemistry.21-00035].

Keywords : Sodium-ion Battery, Tin/Carbon, Cycle Characteristics, Exfoliation

\section{Introduction}

The demand for secondary batteries with high capacity, long life, and low cost have increased in recent years for applications such as electric vehicles and power storage systems. In particular, lithium-ion batteries, which are the representative secondary batteries, require rare metals such as $\mathrm{Li}$ and $\mathrm{Co}$. Therefore, to avoid problems such as resource depletion and price hikes in the future, it is necessary to develop post-lithium-ion batteries of high energy density that can be prepared from abundant and low cost natural resources.

Sodium-ion batteries (SIBs) are promising as next-generation batteries, as reviewed initially by Slater et al. ${ }^{1}$ and recently by Perveen et al.: ${ }^{2}$ elemental sodium has an approximately 500 times larger Clarke number than lithium, and is inexpensive at approximately $1 / 30$ the price of lithium as a carbonate material. ${ }^{1}$ Hard carbons were mainly studied initially as anode materials for SIBs, of which the discharge capacities were reported to be approximately 250 to $350 \mathrm{mAh} \mathrm{g}^{-1} \cdot{ }^{3-8}$ Unlike hard carbons, tin (Sn)-based anode materials (e.g., $\mathrm{Na}_{15} \mathrm{Sn}_{4}$, of which the theoretical capacity is $847 \mathrm{mAh} \mathrm{g}^{-1}$ ) have been actively studied with an aim to increase capacity. ${ }^{9,10}$ Such high capacity holds potential for the future as an alternative to the commonly-used lithium ion battery anode of which the theoretical capacity is $372 \mathrm{mAhg}^{-1}$ (graphite, $\left.\mathrm{LiC}_{6}\right)^{9}$

However, the $\mathrm{Sn} / \mathrm{Na}$ material undergoes large expansion and contraction at the grain scale during the alloying-dealloying reaction throughout the charge-discharge process, and the local volumetric change generates stress in the $\mathrm{Sn} / \mathrm{Na}$ alloy; the volumetric change ratio of expansion to contraction reaches ca. $420 \% .^{1,2}$ Therefore, as the charge-discharge cycle is repeated, the $\mathrm{Sn}$ active materials exfoliate from the current collectors and the capacity is severely degraded. ${ }^{10,11}$

${ }^{\S}$ ECSJ Active Member
To solve the Sn exfoliation problem, some approaches have used material-based composites of Sn with a carbon matrix to ensure a buffer function; tensile, compressive, and shear stresses caused during the charge-discharge process can be relieved by the buffer function of $\mathrm{Sn} /$ carbon complex composites. ${ }^{12-14} \mathrm{Xu}$ et al. initially reported in 2013, a capacity of $295 \mathrm{mAh} \mathrm{g}^{-1}$ at the 15 th cycle with a $\mathrm{Sn} /$ porous-C composite, ${ }^{12}$ and Datta et al. reported $353 \mathrm{mAh} \mathrm{g}^{-1}$ at the 20th cycle for a combination of micro-Sn particles and a carbon material. ${ }^{13} \mathrm{Kim}$ et al. combined porous carbon with nano-Sn particles and achieved a maximum capacity of $674 \mathrm{mAh} \mathrm{g}^{-1}$ with $519 \mathrm{mAh} \mathrm{g}^{-1}$ at the 500th cycle. ${ }^{14}$ Fukunishi et al. next introduced another carbon-combined micro-Sn particle based material to achieve a capacity of $676 \mathrm{mAh} \mathrm{g}^{-1}$ during early cycles, although the capacity gradually and monotonically decreased to ca. 530 $\mathrm{mAhg}^{-1}$ at the 50th charge-discharge cycle, and finally to 470 $\mathrm{mAh} \mathrm{g}^{-1}$ at the 100 th cycle. ${ }^{11}$ Pan et al. used Sn and C-nanosheets and also achieved a capacity of $627 \mathrm{mAh} \mathrm{g}^{-1}$ recently, even after 50 cycles. ${ }^{15}$

Mechanical milling produces finer particles, reduces the crystallite size, and imparts local disorder in the crystal structure (lattice defects) by application of mechanical energy to the particles in the form of friction and mechanical stress. The effects of crystallite size-reduction and disorder have been utilized to improve the functionality of active materials. ${ }^{13,16,17}$ Acetylene black (AB) is a representative conductive material that exhibits (1) electrical conductivity, where the conductive material forms a network through which electrons flow between the current collector and the conductive material, and (2) retention, where the conductive material forms voids that absorb and retain electrolyte solution.

The introduction of $\mathrm{AB}$ as a conductive material could solve the exfoliation problem; the combination of $\mathrm{Sn}$ and $\mathrm{AB}$ by mechanical milling could produce $\mathrm{Sn} / \mathrm{AB}$ composites with stability against the exfoliation, in addition to crystallite size reduction and disorder. 
Mechanical milling fractures $\mathrm{AB}$ particles, so that they can coat the Sn-particle surfaces to produce Sn/AB composites.

Such $\mathrm{Sn} / \mathrm{AB}$ composites were prepared to produce Sn-based electrodes with a more stabilized structure that was expected to reduce the influence of the volumetric change and prevent exfoliation of the active material. The $\mathrm{Sn} / \mathrm{AB}$ composites in this work were produced by mechanical milling for various times in an attempt to achieve battery cycle characteristics that were as well as the highest of those reported previously. Some cause-effect relationships in capacity deterioration were also examined for the $\mathrm{Sn} / \mathrm{AB}$ composites with respect to the particle size distributions and the specific surface areas. The crystal structures were also investigated using X-ray diffraction (XRD) and the surfaces of the powdered composites and the cross sections of electrodes were observed using field emission scanning electron microscopy (FESEM).

\section{Preparation of Electrodes}

\subsection{Raw materials}

Sn powder (Special grade, Nacalai Tesque Inc.) was used as the active material for the anode material. AB powder (Denka Black, Denka Co., Ltd.) was used to synthesize the $\mathrm{Sn} / \mathrm{AB}$ composite material.

\subsection{Two-step addition of $A B$}

To synthesize an anode composite material for an SIB, AB was added to Sn using two steps. Figure 1 shows a schematic diagram of the compounded $\mathrm{Sn} / \mathrm{AB}$ composite. In the first step, $\mathrm{AB}$ was added as a coating material onto $\mathrm{Sn}$ particles to mitigate the volumetric change by the $\mathrm{Sn} / \mathrm{Na}$ alloying process in the charge-discharge cycle, and suppress exfoliation of the active materials from the current collector. This $\mathrm{AB}$ is referred to as coating $\mathrm{AB}$ in the following, and the coating $\mathrm{AB}$ was prepared by mechanical milling as explained in the next section. In the second step, $\mathrm{AB}$ was added as a conductive material to form networks through which electrons flow between the current collector and Sn, and to form voids in which the electrolyte solution is retained. This $\mathrm{AB}$ is referred to as conductive $\mathrm{AB}$ in the following.

\subsection{Electrode preparation procedure}

Figure 2 shows the procedure used to prepare the $\mathrm{Sn} / \mathrm{AB}$ composite. Sn and $\mathrm{AB}$ for coating were first mechanically milled, whereby agglomerated $\mathrm{AB}$ powders could be disintegrated into small particles that coat the Sn particles A ball milling device (Fritsch Pulverisette 6) equipped with a cylindrical zirconia $\left(\mathrm{ZrO}_{2}\right)$ container $(66 \mathrm{~mm}$ diameter, $80 \mathrm{~mL})$ was used, and $10 \times 10 \mathrm{~mm}$ diameter and $20 \times 5 \mathrm{~mm}$ diameter $\mathrm{ZrO}_{2}$ balls were placed into the

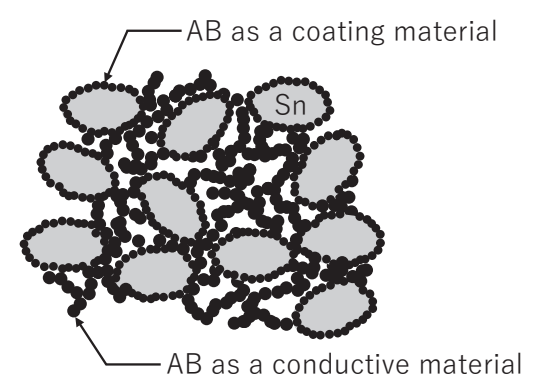

Figure 1. Schematic diagram of the compounded Sn (large gray ellipses)/AB (small black filled circles) composite where the $\mathrm{AB}$ particles are coated on the surface of Sn particles. Another type of $\mathrm{AB}$ particles (larger black filled circles) connect the AB-coated $\mathrm{Sn}$ particles and act as both a conductive agent and for retention of the electrolyte.

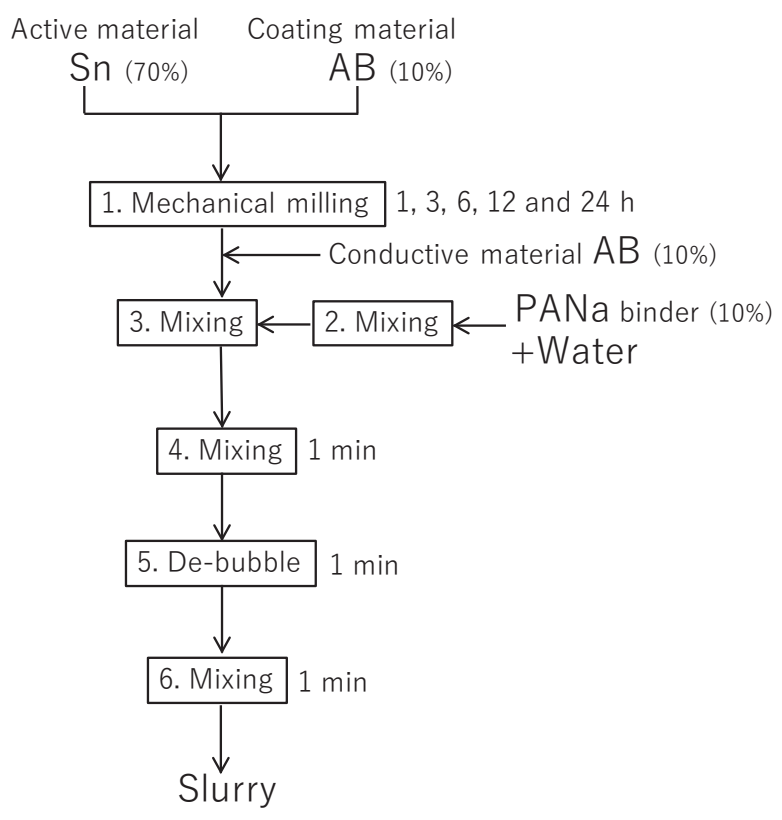

Figure 2. Procedure for preparation of the $\mathrm{Sn} / \mathrm{AB}$ composite. The mass ratio between $\mathrm{Sn}$, coating $\mathrm{AB}$, conductive $\mathrm{AB}$, and $\mathrm{PANa}$ is $7: 1: 1: 1$.

container. The mass mixing ratio of Sn powder and the coating $\mathrm{AB}$ powder was fixed at 7:1 for each of the $\mathrm{Sn} / \mathrm{AB}$ samples. The mixture was rested for a $5 \mathrm{~min}$ interval between $15 \mathrm{~min}$ of milling at $300 \mathrm{rpm}(3.3 \mathrm{G})$ to avoid excessive temperature increase. The milling-rest processes were continued to give total processing times of $1,3,6,12$, and $24 \mathrm{~h}$. The composite powders are collectively referred to as $\mathrm{Sn} / \mathrm{ABs}$, and each type is referred to as, for example, $\mathrm{Sn} / \mathrm{AB}_{-} 1 \mathrm{~h} .0 .154 \mathrm{~g}$ of an aqueous solution of binder containing 16.2 wt\% sodium polyacrylate (PANa; Daiichi Kogyo Seiyaku Co., Ltd. M.W.: $140,000 \mathrm{~g} \mathrm{~mol}^{-1}$ ) was weighed and added to $0.75 \mathrm{~mL}$ of pure water in a $10 \mathrm{~mL}$ polypropylene (PP) container, and then stirred with a defoaming kneader (AR-100, Thinky Co., Ltd.) to obtain a diluted aqueous solution. $0.200 \mathrm{~g}$ of the $\mathrm{Sn} / \mathrm{AB}$ composite and $0.025 \mathrm{~g}$ of conductive $\mathrm{AB}$ were then weighed and mixed, and then placed into the PP container with the PANa solution. As a result, the mass ratio of $\mathrm{Sn}$ to coating $\mathrm{AB}$ to conductive $\mathrm{AB}$ to $\mathrm{PANa}$ was $7: 1: 1: 1$. Finally, the mixture was stirred, defoamed, and again stirred for $1 \mathrm{~min}$ to obtain a slurry electrode material. The slurry was applied to a $20 \mu \mathrm{m}$ thick aluminum foil current collector using an applicator, dried under vacuum at $130^{\circ} \mathrm{C}$ for $4 \mathrm{~h}$, and then punched into $12 \mathrm{~mm}$ diameter electrodes. No pressing treatment was applied during the fabrication of coin cells to improve the flowability for expansion and contraction of the active materials. The amount of active material on the current collector was adjusted within the range of $1.0 \pm 0.2 \mathrm{mg} \mathrm{cm}^{-2}$.

\section{Characterization of $\mathrm{Sn}$ and Sn/AB Composites}

The particle size distributions were measured by the wet method with a laser particle size analyzer (LA-920, Horiba, Ltd.). The median particle diameter of a sample before and after mechanical milling were then calculated based on the measured distributions. The specific surface areas were calculated using isotherm adsorption curves of a $\mathrm{He}+\mathrm{N}_{2}$ mixed gas by the Brunauer-Emmett-Teller (BET) method with a surface area analyzer (Macsorb HM-1201, Mountech Co., Ltd.). The relationship between the mechanical milling duration time and the crystal structure was investigated using XRD (Ultima IV, Rigaku Co., Ltd.) with $\mathrm{CuK} \alpha$ radiation at an output of $40 \mathrm{kV}$ and $20 \mathrm{~mA}$, a sampling interval of $0.02^{\circ}$, and a scan speed of $2^{\circ} \mathrm{min}^{-1}$ in the range of $10-80^{\circ}$. The surface of the 
powdered composites and cross sections of the electrodes were observed using FE-SEM (JSM-7001F, JEOL Ltd.) at an accelerating voltage of $5 \mathrm{kV}$ or $10 \mathrm{kV}$. The electrode samples were embedded in a thermosetting conductive resin and polished for FE-SEM cross sectional observations.

Batteries for charge-discharge tests were fabricated with stainless steel (SUS316L) 2032 type coin cells, assembled in a glove box with the dew point kept below $-70^{\circ} \mathrm{C}$. The fabricated electrodes were used as working electrodes and sodium metal was used as the counter electrode. Two pieces of $16 \mathrm{~mm}$ diameter Celgard \#2400 were used as separators, and a $14 \mathrm{~mm}$ diameter glass fiber filter (Advantec GB-100R) was sandwiched between the two separators. A SUS316L steel spacer and a spring were arranged on the counter electrode side. $1.0 \mathrm{~mol} \mathrm{dm}^{-3} \mathrm{NaPF}_{6}$ dissolved in ethylene carbonate (EC) and diethylene carbonate (DEC) (49:49 v/v, Kishida Chemical Co., Ltd.) with 2 vol\% of fluoroethylene carbonate (FEC; Kishida Chemical Co., Ltd.) was used as the electrolyte solution. The coin cell batteries were stored at $25^{\circ} \mathrm{C}$ and were charged and discharged using a charge-discharge tester (HJ1001SD8, Hokuto Denko) under constant current (CC) conditions. The current was set at $50 \mathrm{~mA} \mathrm{~g}^{-1}$, except for the first cycle, which was set at $25 \mathrm{~mA} \mathrm{~g}^{-1}$. Current density and specific capacity were calculated based on the mass of Sn.

The cutoff voltages were $0.005 \mathrm{~V}$ for the charge process and $0.650 \mathrm{~V}$ for the discharge process with a rest time of 3 min inserted between the charge and discharge periods. The sample number of batteries prepared for each test was $n=3$, and the sample data with the least discharge capacity degradation during cycling were used for evaluation.

\section{Experimental Results and Discussion}

\subsection{Morphological changes of $\mathrm{Sn}, \mathrm{AB}, \mathrm{Sn} / \mathrm{ABs}$ by mechanical milling}

Figure 3 shows the change in the median particle diameter and the specific surface area of $\mathrm{Sn} / \mathrm{ABs}$ for various milling times. The median particle diameters of $\mathrm{Sn}$ and $\mathrm{AB}$ before compounding were 13.8 and $4.72 \mu \mathrm{m}$, respectively. The median particle diameter of Sn/ AB_1h was $31.2 \mu \mathrm{m}$, which was larger than $13.8 \mu \mathrm{m}$ for the Sn-only powder. This is caused partly by consolidation of the Sn particles themselves because $\mathrm{Sn}$ is a relatively soft metal, and partly by the adhesion of larger-diameter $\mathrm{AB}$ agglomerates. The median particle diameter of $\mathrm{Sn} / \mathrm{AB}_{-} 3 \mathrm{~h}$ decreased to as small as $11.6 \mu \mathrm{m}$ along with the progress $\mathrm{AB}$ agglomerate disintegrating. The median particle diameters for $\mathrm{Sn} / \mathrm{AB}_{-} 6 \mathrm{~h}, \mathrm{Sn} / \mathrm{AB}_{-} 12 \mathrm{~h}$, and $\mathrm{Sn} / \mathrm{AB}_{-} 24 \mathrm{~h}$ were 8.62, 8.78 , and $10.4 \mu \mathrm{m}$, respectively, showing no significant change. The specific surface areas of the $\mathrm{Sn}$ and $\mathrm{AB}$ raw materials were 0.20 and

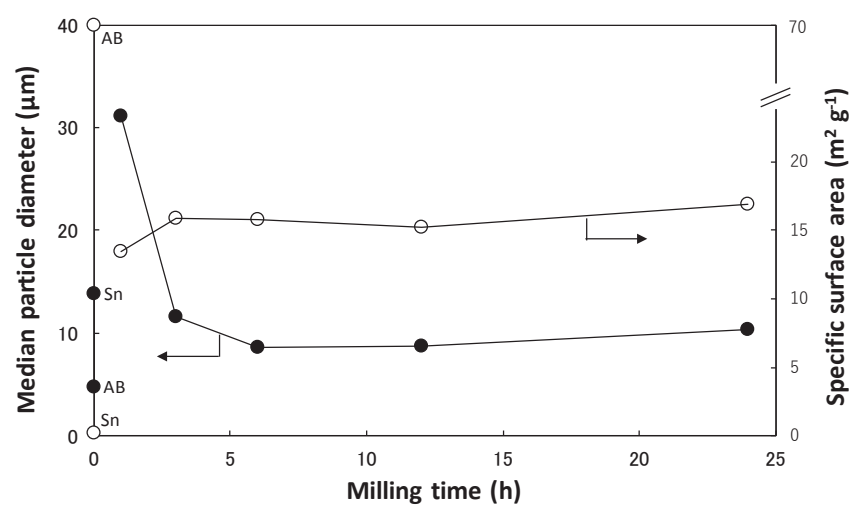

Figure 3. Median particle diameter $\left(D_{50}\right)$ and specific surface area of the $\mathrm{Sn} / \mathrm{AB}$ composites as a function of the mechanical milling time. The median particle diameters of $\mathrm{Sn}$ and $\mathrm{AB}$ were 13.8 and $4.72 \mu \mathrm{m}$, respectively. The specific surface areas of $\mathrm{Sn}$ and $\mathrm{AB}$ were $0.20 \mathrm{~m}^{2} \mathrm{~g}^{-1}$ and $70.1 \mathrm{~m}^{2} \mathrm{~g}^{-1}$, respectively.
$70.1 \mathrm{~m}^{2} \mathrm{~g}^{-1}$, respectively. The surface areas for $\mathrm{Sn} / \mathrm{AB}_{-} 1 \mathrm{~h}, \mathrm{Sn} /$ AB_6h, and Sn/AB_24h were increased gradually to $13.5,15.8$, and $16.9 \mathrm{~m}^{2} \mathrm{~g}^{-1}$, respectively. Similarly to the median particle diameter, the specific surface area changed significantly from milling time of $1 \mathrm{~h}$, although there was no significant change for milling times of $3 \mathrm{~h}$ or more.

Figure 4 shows particle size distributions and SEM images for $\mathrm{Sn}, \mathrm{AB}$ and Sn/ABs. In Fig. 4(a), the distribution of raw $\mathrm{Sn}$ is unimodal with a peak near $15 \mu \mathrm{m}$. Figure 4(b) shows the bimodal distribution of the raw $\mathrm{AB}$ particles with two peaks at 0.51 and $5.87 \mu \mathrm{m}$. The left peak is related to the particle size distribution of the $A B$ primary particles, while the right peak is that for $A B$ agglomerates of $\mathrm{AB}$ particles. The sub-micrometer $\mathrm{AB}$ particles corresponding to the left peak are considered to work as the $A B$ particles for coating of the Sn particles.

On the other hand, the near ten-micrometer $\mathrm{AB}$ agglomerates corresponding to the right peak are considered to work as conductive $\mathrm{AB}$ in the fabricated electrode material. The median particle diameters of the $\mathrm{AB}$ primary particles is ca. $1 / 25$ of the $\mathrm{Sn}$ particles $(13.8 \mu \mathrm{m})$. The strength of the $\mathrm{AB}$ agglomerates against disintegrating in the mechanical milling process is much weaker than that of Sn particles; therefore, the AB particles are expected to cover the Sn particles during this process. While $\mathrm{Sn} / \mathrm{AB}_{-} 1 \mathrm{~h}$ has two peaks related to the particle size of $\mathrm{AB}$ (Fig. 4(c)), the $\mathrm{Sn} / \mathrm{AB}_{-} 3 \mathrm{~h}$, Sn/AB_6h, Sn/AB_12h, and Sn/AB_24h powders (Figs. 4(d)-(g)) have unimodal distributions. In addition, the distribution peak was sharpened as the milling time was increased to $3 \mathrm{~h}$, but remained unchanged for milling times of $6 \mathrm{~h}, 12 \mathrm{~h}$, and $24 \mathrm{~h}$. The SEM images show the $\mathrm{Sn}$ particles are rounded, whereas the $\mathrm{AB}$ particles are aggregated. The Sn/ABs reflect the shape of the Sn particles, no apparent change in shape was evident, particularly for milling times of $3 \mathrm{~h}$ or more. Figure 5 shows magnified SEM images of the raw $\mathrm{Sn}$ and $\mathrm{Sn} / \mathrm{AB} \_6 \mathrm{~h}$ particles. The rounded $\mathrm{Sn}$ particles are covered with fine submicron-sized $\mathrm{AB}$ particles after mechanical milling.

Figure 6(a) shows XRD patterns of the $\mathrm{Sn} / \mathrm{ABs}$ for the various milling times. No significant change was observed in the XRD diffraction patterns, which suggests that the crystal structure did not change from the mechanical milling process. Figure 6(b) shows magnified views of the peaks around $30.6^{\circ}$ from the (200) plane and that around $32.0^{\circ}$ from the (101) plane; both peaks are slightly shifted to the low angle side with an increase of the milling time. It is considered that the $\mathrm{Sn}$ atomic arrangement is partly disturbed by the centrifugal force associated with rotation and revolution during mechanical milling, which results in the decrease of the crystallite size and the formation of lattice defects, which is reported to slightly widen the lattice spacing. ${ }^{18}$ Therefore, the lattice spacing is widened and the peak is shifted to the low angle side. On the other hand, the full width at half maximum (FWHM) tends to increase and the crystallite size decreases as the milling time increases. The crystallite size for each of the milling times is shown in Table S1 (Supporting Information).

\subsection{Charge and discharge tests}

Figure 7 shows charge-discharge curves up to the 10th cycle for SIBs with the $\mathrm{Sn}$ and $\mathrm{Sn} / \mathrm{ABs}$ used as the working electrodes. The charge-discharge curves of the coin cell fabricated with $\mathrm{AB}$ and without $\mathrm{Sn}$ is shown in Fig. S1 (Supporting Information). The charge (sodiation) capacity was ca. $400 \mathrm{mAh} \mathrm{g}^{-1}$ at the 1 st cycle, which then increased to ca. $600 \mathrm{mAh} \mathrm{g}^{-1}$ in the 10 th cycle. However, there were unstable charge capacity changes during the 1 st to 3 rd cycles, which was considered to be caused by the structural changes associated with the repeated alloying and dealloying reactions of $\mathrm{Sn}$ and $\mathrm{Na}^{19}$ Plateaus were commonly observed during the 1st charge cycle; plateaus occurred near $0.1 \mathrm{~V}$ with the Sn-only electrode (Fig. 7(a)), and around $0.4 \mathrm{~V}$ with the $\mathrm{Sn} / \mathrm{AB}$ composites (Figs. 7(c) to 7(f)). Fukunishi et al. ${ }^{11}$ explained 

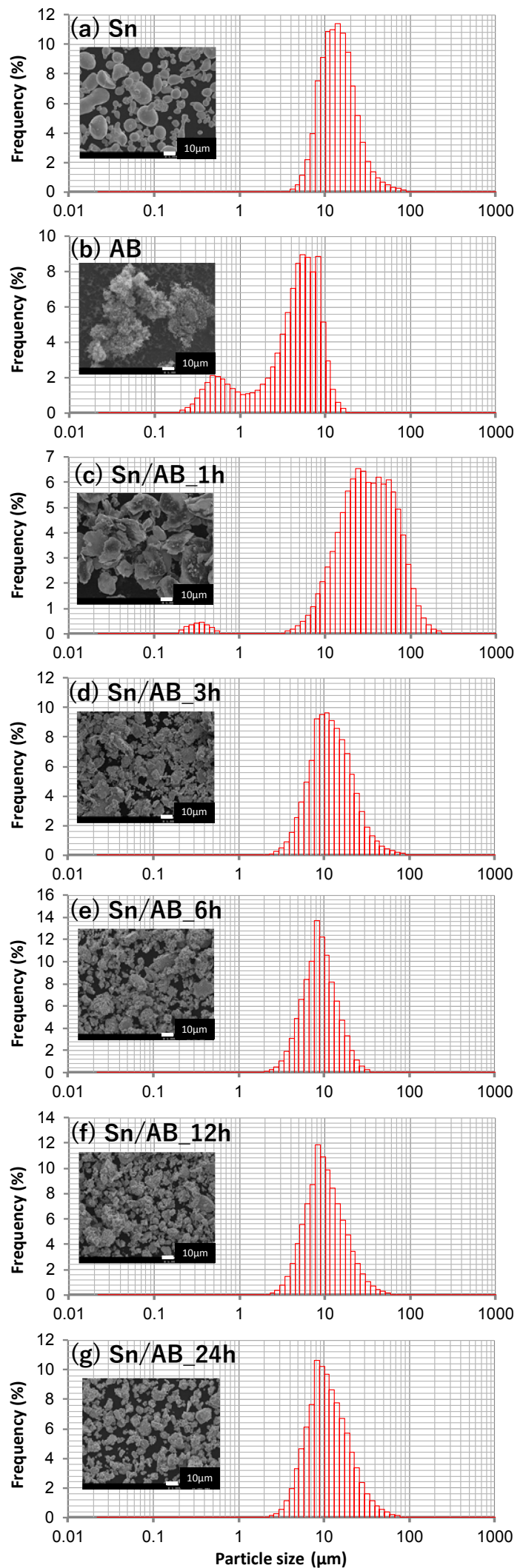

Figure 4. Particle size distributions and SEM images of the raw $\mathrm{Sn}$, raw $\mathrm{AB}$, and $\mathrm{Sn} / \mathrm{AB}$ composite powders; (a) Sn, (b) AB, (c) Sn/ AB_1h, (d) Sn/AB_3h, (e) Sn/AB_6h, (f) Sn/AB_12h, and (g) Sn/ AB_24h.

the latter plateau as follows. The conductive $\mathrm{AB}$ particles, which have large specific surface areas and are present around the $\mathrm{Sn}$ particle, absorb the binder on the surface of the Sn particles, which results in a reduction of the binder. This phenomenon causes a reduction of the resistance, which results in the plateaus. In this

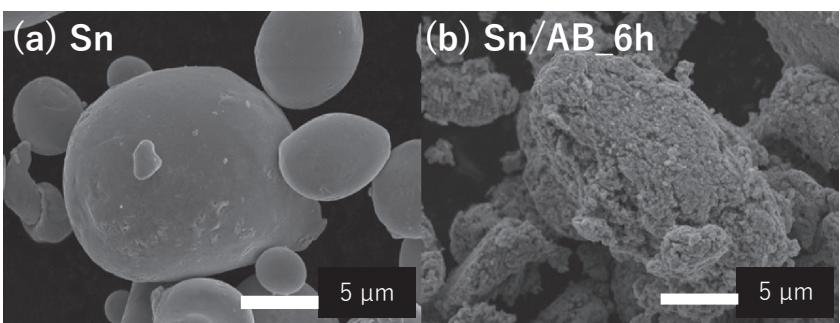

Figure 5. SEM images of (a) raw $\mathrm{Sn}$ and (b) Sn/AB_6h particles.

(a)
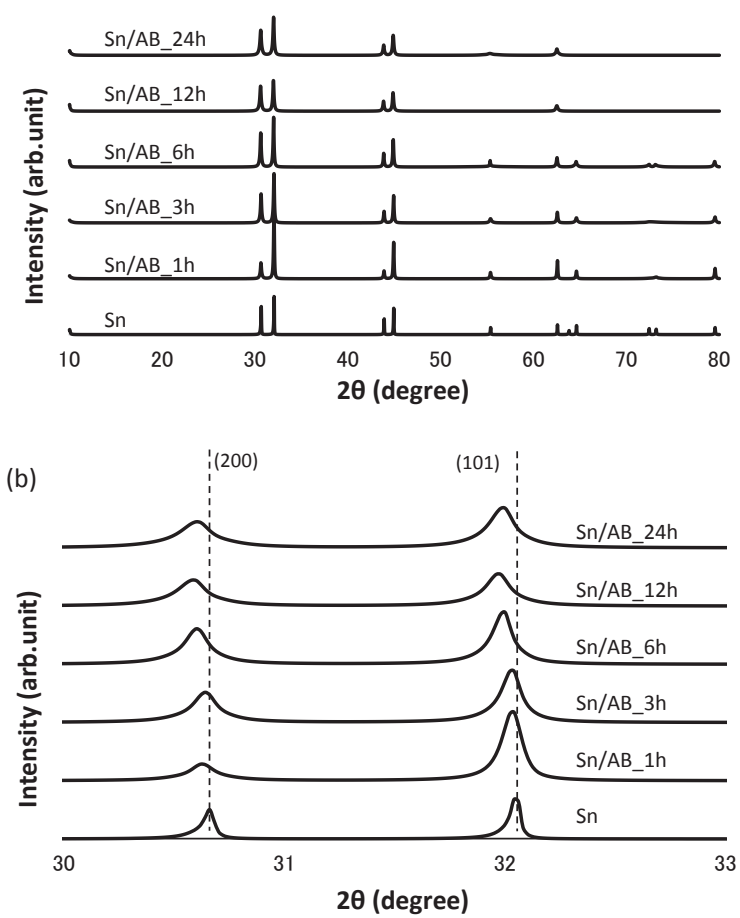

Figure 6. XRD patterns of $\mathrm{Sn}$ and $\mathrm{Sn} / \mathrm{AB}$ composites for various milling times. (a) General XRD patterns for $2 \theta$ from 10 to $80^{\circ}$. (b) Magnified XRD patterns for $2 \theta$ from 30 to $33^{\circ}$; the peaks around $30.6^{\circ}$ is from the (200) plane and that around $32.0^{\circ}$ from the (101) plane are shown separately.

study, a plateau around $0.4 \mathrm{~V}$ was observed for milling times of $3 \mathrm{~h}$ or more; therefore, it is considered that there are many more pulverized $\mathrm{AB}$ agglomerates around the Sn particles.

The charge curves for the Sn-only and $\mathrm{Sn} / \mathrm{ABs}$ electrodes after the 2nd cycle showed similar patterns (Figs. 7(a) to 7(f)). In the charge-discharge tests, better performance would appear in the early cycles, whereas the performance would later be degraded after repeated charge-discharge processes. The performance in the early cycles thus exhibits the potential abilities of these candidate materials with Sn. Overall, the Sn/ABs exhibit some properties similar to Sn, regardless of the presence of $\mathrm{AB}$ particles around the Sn particles.

\subsection{Cycle characteristics of $\mathrm{Sn}$ and $\mathrm{Sn} / \mathrm{AB}$ samples}

Figure 8 shows the cycle characteristics of the prepared batteries, in which Sn-only and Sn/ABs with different milling times were used. The discharge capacity tended to increase from the 1 st to approximately the 15th cycle, regardless of the milling time. In later cycles, the discharge capacities with Sn-only, Sn/AB_lh, and Sn/ AB_3h decreased gradually after approximately $20-35$ cycles. On the other hand, Sn/AB_6h, Sn/AB_12h, and Sn/AB_24h exhibited stable cycle characteristics up to 50 cycles, i.e., the final cycle number in this work, the discharge capacities at the 50th cycle of 

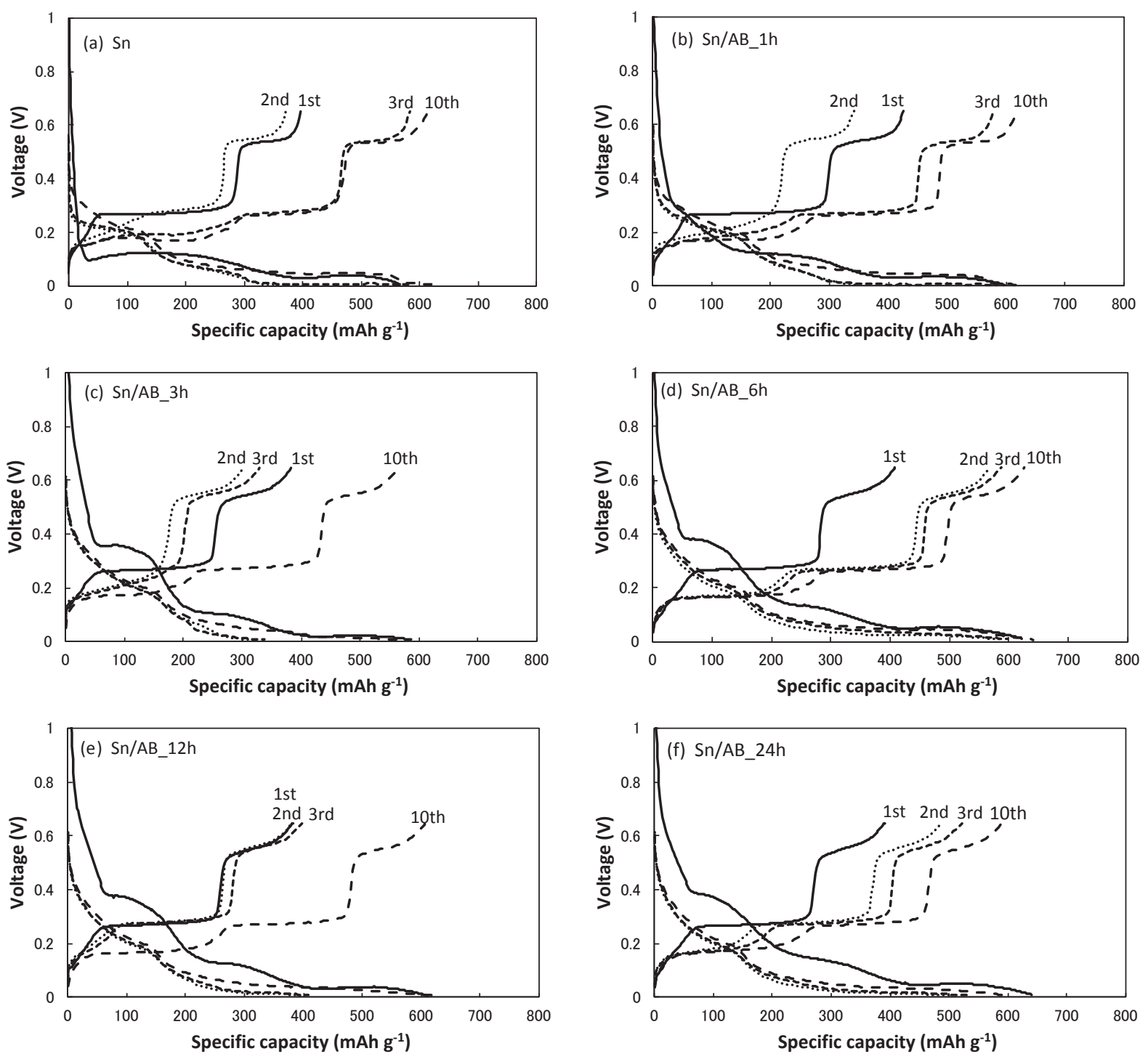

Figure 7. Charge-discharge curves for SIBs with Sn-only and Sn/AB electrodes up to the 10th cycle; (a) Sn-only, (b) Sn/AB_lh, (c) Sn/ AB_3h, (d) Sn/AB_6h, (e) Sn/AB_12h, and (f) Sn/AB_24h.

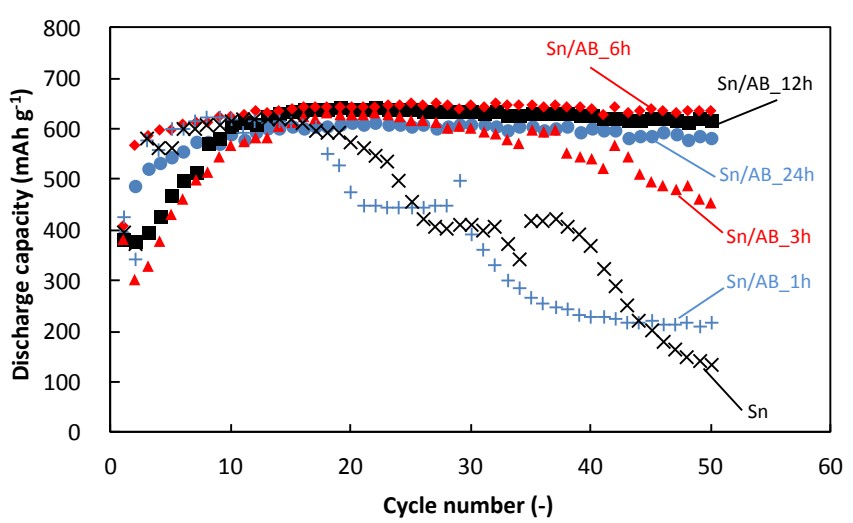

Figure 8. Cycle characteristics of Sn-only and Sn/ABs electrodes prepared with various milling times.

which were 635,619 , and $584 \mathrm{mAh}^{-1}$, respectively. For $\mathrm{Sn} /$ AB_6h, Sn/AB_12h, and Sn/AB_24h, the Sn particles are suitably coated with the $A B$ particles, which mitigates the expansion and contraction caused by $\mathrm{Sn}-\mathrm{Na}$ alloying-dealloying, and suppresses exfoliation of the active materials from the current collector. This suppression of structural decay in the composites leads to better cycle characteristics of the batteries.
Although previous studies on carbon composites with micrometer-sized Sn have not yet obtained capacities greater than 600 $\mathrm{mAhg}^{-1}$ after 50 cycles, ${ }^{11,13}$ the present work employed the micrometer-sized Sn with a distribution peak near $15 \mu \mathrm{m}$, which was similar to the previous studies, ${ }^{11,13}$ and a higher discharge capacity of $635 \mathrm{mAh} \mathrm{g}^{-1}$ was achieved after 50 cycles. The reason for this is considered to be that the $\mathrm{AB}$ particles used in the previous study ${ }^{11}$ only worked as a conductive material, of which particle size was several micrometers, which was similar to the size of the conductive $\mathrm{AB}$ particles used in the present study. The only difference was the use of the approximately sub-micrometer coating AB particles produced by mechanical milling in this study.

\subsection{Cycle characteristics and coulombic efficiencies of Sn- only and the Sn/AB composites}

Figure 9 shows the cycle characteristics and coulombic efficiencies of the batteries prepared using Sn-only and Sn/AB_6h. The coulombic efficiencies of both Sn-only and $\mathrm{Sn} / \mathrm{AB}_{-} 6 \mathrm{~h}$ were slightly less than $70 \%$ in the 1 st cycle, which increased up to ca. $97 \%$ in the 2nd cycle, and then remained constant in the subsequent cycles. The low coulombic efficiency in the first cycle is considered to be due to the formation of a solid electrolyte interphase (SEI) on the Sn anode. ${ }^{19}$

The cycle characteristics of Sn/AB_6h were much better than those of Sn-only, although the coulombic efficiencies were almost 


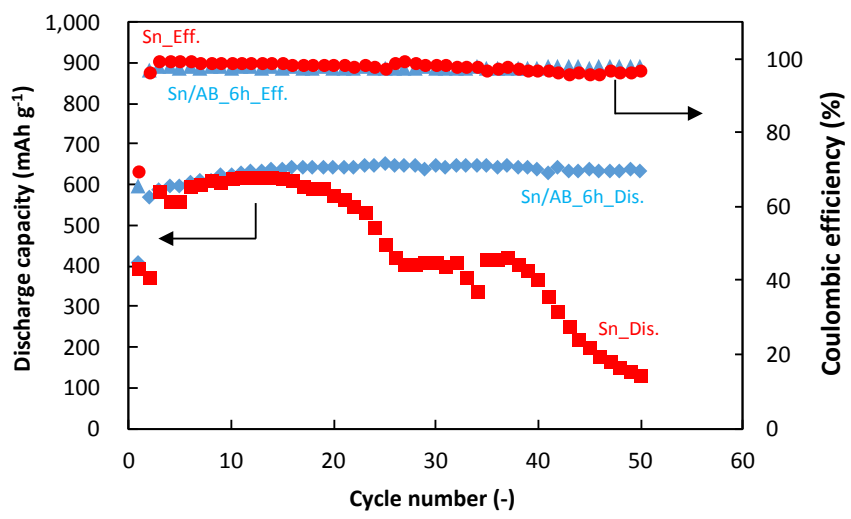

Figure 9. Cycle characteristics and coulombic efficiency of SIBs using Sn-only and Sn/AB_6h electrodes.

equivalent. This indicates that in both Sn-only and Sn/AB_6h, Na ions are alloyed with Sn during charge and are de-alloyed from $\mathrm{Sn}$ during discharge, whereby the ions return to the opposite electrode. However, a rapid drop in capacity was observed with Sn-only. It is thus considered that the insertion reaction of $\mathrm{Na}$ into $\mathrm{Sn}$ during charge is likely to be obstructed as the number of cycles increase.

\subsection{Discharge capacities at maximum and those after 50 cycles}

Figure 10 shows the maximum discharge capacities and the discharge capacities after 50 cycles for Sn-only and all the $\mathrm{Sn} / \mathrm{ABs}$ as a function of the median particle diameter and the specific surface area. The maximum discharge capacities in Fig. 10(a) are almost constant, regardless of the median particle diameter in Sn-only or the Sn/ABs, and similarly, in Fig. 10(b), the maximum discharge capacity is almost constant, regardless of the specific surface area. On the other hand, Fig. 10(c) shows the capacity after 50 cycles,
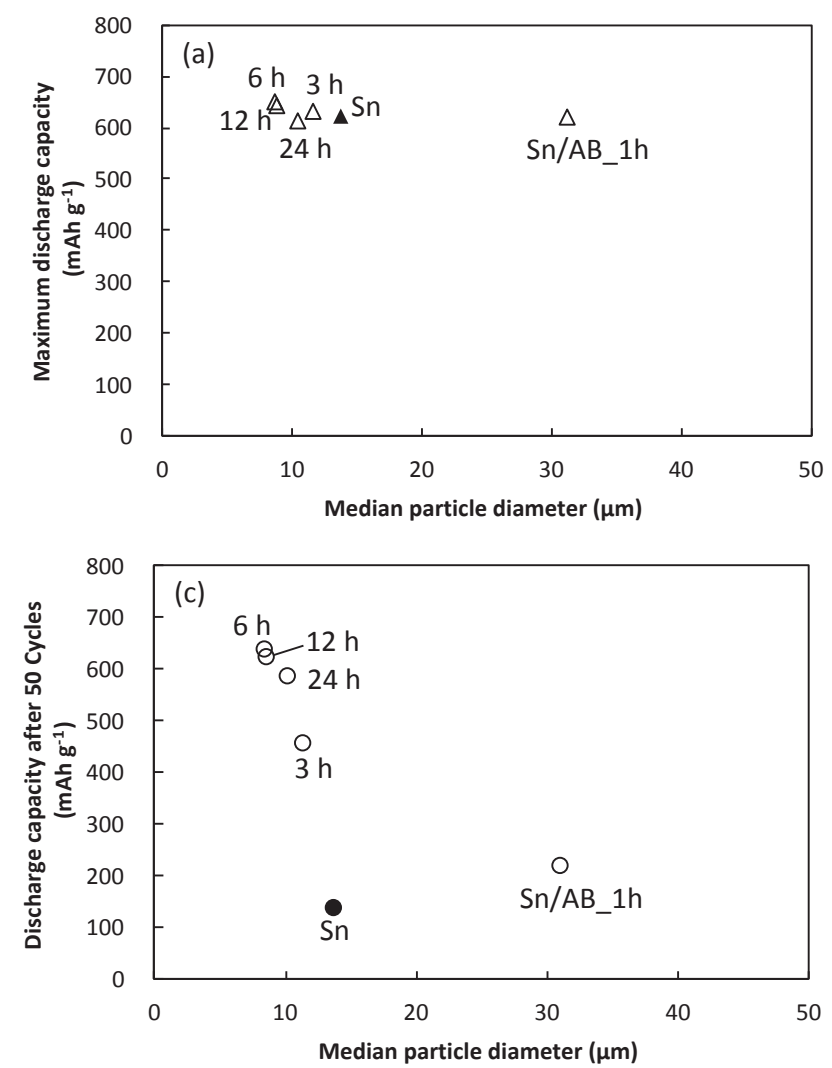

which tended to increase as the median particle diameter decreased. In addition, Fig. 10(d) shows the capacity after 50 cycles, which tended to increase as the specific surface area increased. As the sizes of the composite particles decrease, the number of voids per unit volume increases, which may reduce the degradation by expansion and contraction. In addition, the reaction-site surface areas per unit volume increase with the specific surface areas of the composites. These two effects may thus reduce the effect of expansion and contraction during the alloying-dealloying of $\mathrm{Sn}$ and $\mathrm{Na}$ in the charge-discharge processes.

\subsection{Observation of surface and cross section of electrodes}

Figure 11 shows SEM images of the Sn-only and Sn/AB_6h electrode surfaces before charge-discharge and those after 50 charge-discharge cycles. Both Sn-only and Sn/AB_6h have a uniform appearance before the charge-discharge cycle, although there are some particulates on the surface. In contrast to these, after 50 cycles, Sn particle growth was evident and the gaps between the particles become wider. In particular, the gaps in Sn-only were much wider than those in Sn/AB_6h, and there was some evidence of exfoliation of the particles. The observation of exfoliation is considered to support the inference that, as stated in Section 4.4, the amount of Sn to be alloyed with $\mathrm{Na}$ decreases at the $\mathrm{Sn}$ electrode sites with an increase in the cycles, so that the capacity deteriorates.

Figure 12 shows SEM images of cross sections of the Sn-only and $\mathrm{Sn} / \mathrm{AB}$-6h electrodes before the charge-discharge cycles. In the Sn-only electrode, a ca. $3 \mu \mathrm{m}$ thick Sn layer covered the Al foil current collector with no gaps (Fig. 12(a)). On the other hand, in the $\mathrm{Sn} / \mathrm{AB}$-6h electrode, the $\mathrm{Sn} / \mathrm{AB}$ composite shown by the white layer is stacked irregularly, and the black-colored conductive ABs (microparticles not disintegrated by mechanical milling) are inserted in the Sn/AB composite layer (Fig. 12(b)).

In both the Sn-only and Sn/AB_6h electrodes, the Sn particles themselves undergo isotropic expansion and contraction during
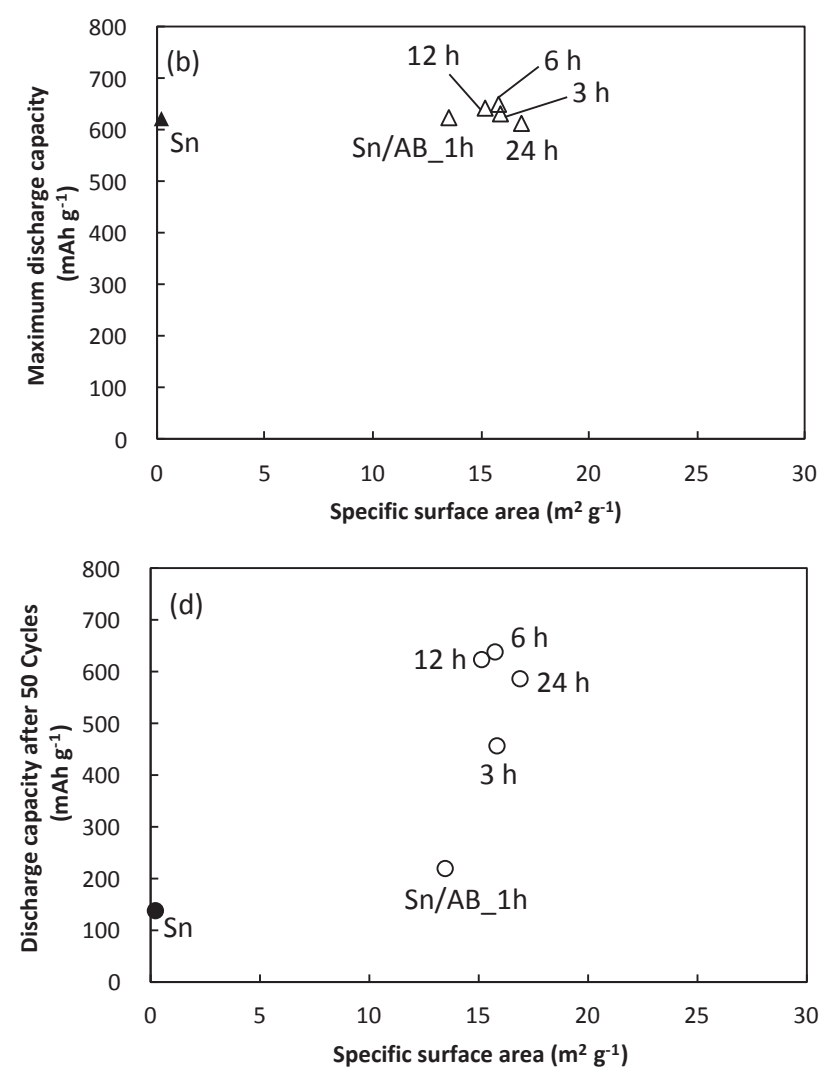

Figure 10. Discharge capacities of the Sn-only and Sn/AB electrodes. (a) Maximum discharge capacities with respect to the median particle diameter. (b) Maximum discharge capacities with respect to the specific surface area. (c) Discharge capacities after 50 cycles as a function of the median particle diameter. (d) Discharge capacities after 50 cycles as a function of the specific surface area. 


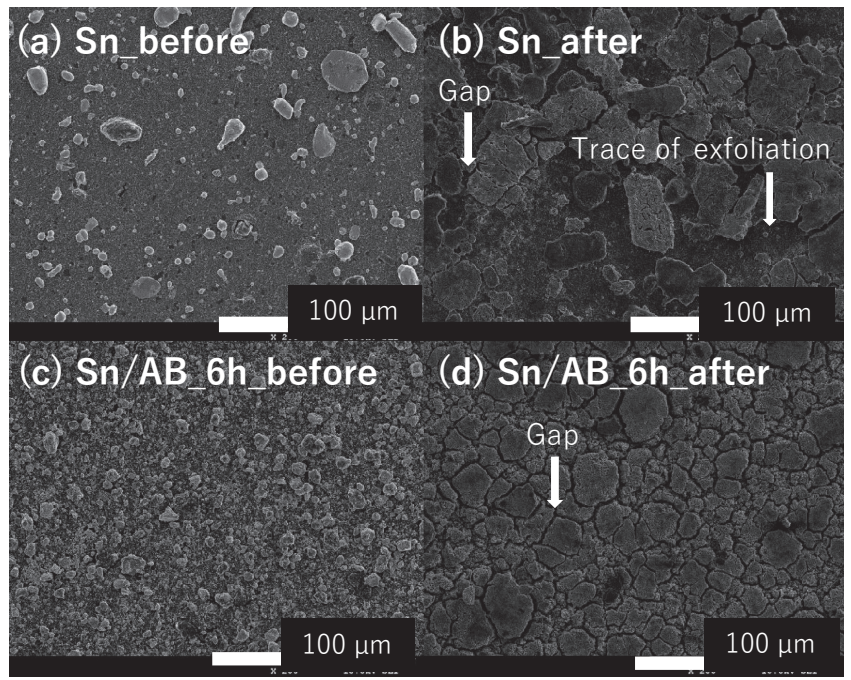

Figure 11. SEM images of (a) Sn-only ( $\mathrm{Sn}$ and conductive $\mathrm{AB}$ particles) before charge-discharge, (b) Sn-only after 50 chargedischarge cycles, (c) Sn/AB_6h (Sn with AB coating and conductive $\mathrm{AB}$ particles) before charge-discharge, and (d) $\mathrm{Sn} / \mathrm{AB} \_6 \mathrm{~h}$ after 50 charge-discharge cycles.

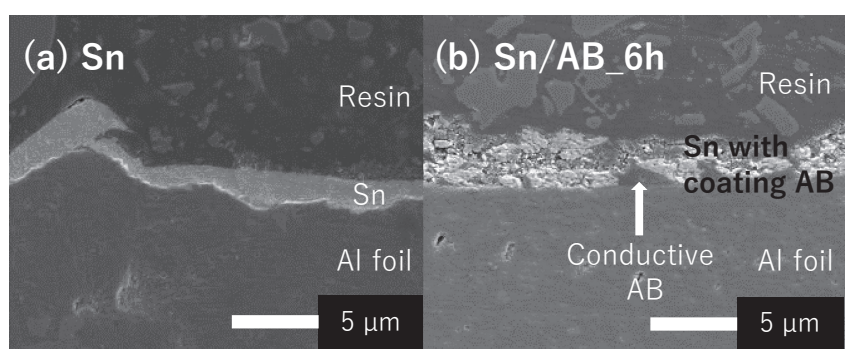

Figure 12. SEM cross section images of Sn-only and Sn/AB_6h electrodes before the charge-discharge cycles. (The resin layer, $\mathrm{Sn}$ or $\mathrm{Sn} / \mathrm{AB}$ layer, and $\mathrm{Al}$ foil layer can be seen from top to bottom).

alloying with $\mathrm{Na}$. In the case of Fig. 12(a), in which the Sn layer continuously covers the Al current collector, the strain in the direction parallel to the electrode surface is accumulated over the electrode surface. As a result, large displacements occur in the $\mathrm{Sn}$ layer, and exfoliation tends to occur. In contrast, the $\mathrm{Sn} / \mathrm{AB}$ composites are attached to the Al current collector in the form of irregularly discontinuous layers of unevenly shaped particles. Therefore, the strain parallel to the electrode surface direction of the electrode is not accumulated across the multiple $\mathrm{Sn} / \mathrm{AB}$ composite particles, and exfoliation will not readily occur. This results in suppression of the capacity degradation in the $\mathrm{Sn} / \mathrm{ABs}$ after repeated charge-discharge cycles.

\section{Conclusion}

SIBs were fabricated as coin cells using an electrode in which micrometer-sized Sn particles were compounded with sub-micrometer $\mathrm{AB}$ particles by mechanical milling, and were electrochemically evaluated. The following results were confirmed.

Various mechanical milling times from 1 to $24 \mathrm{~h}$ resulted in changes to the median particle diameters and specific surface areas of the electrode materials, although they became almost constant with milling times of $3 \mathrm{~h}$ or more. The peak width of the particle size distribution became sharper as the milling time increased up to $6 \mathrm{~h}$, and then became almost unchanged for more than $6 \mathrm{~h}$ milling. Charge-discharge tests of the Sn-only or $\mathrm{Sn} / \mathrm{ABs}$ electrodes up to 50 cycles indicated a milling time of $6 \mathrm{~h}$ or more resulted in more stable cycle characteristics. For example, the cell with the Sn/AB_6h electrode exhibited a discharge capacity of $635 \mathrm{mAh} \mathrm{g}^{-1}$, in contrast to $135 \mathrm{mAh} \mathrm{g}^{-1}$ for the cell with the Sn-only electrode. The effect of $\mathrm{AB}$ coating was insufficient when the milling time was $3 \mathrm{~h}$ or less. In contrast, milling times of $6 \mathrm{~h}$ or more resulted in sufficient coating of the sub-micrometer $\mathrm{AB}$ particles on the Sn particles, which suppressed the effect of the structural change during the alloyingdealloying processes of $\mathrm{Sn}$ with $\mathrm{Na}$.

The median particle diameter and specific surface area were almost constant after $6 \mathrm{~h}$ milling; therefore, no significant change would be expected for milling treatment greater than $24 \mathrm{~h}$. Therefore, the charge-discharge characteristics would also show no significant change for greater milling times.

SEM observations of the electrode cross sections indicated that exfoliation of the Sn particles that function as active materials in the electrode was avoided in the $\mathrm{Sn} / \mathrm{AB}$ electrodes milled for $6 \mathrm{~h}$ or more due to the formation of a layer constituted of discontinuouslyformed and irregularly-shaped conductive- $\mathrm{AB}$ particles. This resulted in the suppression of capacity degradation after repeated charge-discharge cycles.

In the future, considering the practical use of the SIBs proposed here, we plan to further develop these batteries with an aim to increase the capacity and stability of the cycle characteristics through charge-discharge tests of more than 50 cycles.

\section{Supporting Information}

The Supporting Information is available on the website at DOI: https://doi.org/10.5796/electrochemistry.21-00035.

\section{Acknowledgments}

The authors are grateful to Daiichi Kogyo Seiyaku Co., Ltd. for supplying the PANa binder. We would like to thank Dr. Yoshihiko Nomura, the executive advisor of Mie Prefecture Industrial Research Institute and an honorary professor of Mie University, for useful advice.

\section{References}

1. M. D. Slater, D. Kim, E. Lee, and C. S. Johnson, Adv. Funct. Mater, 23, 947 (2013).

2. T. Perveen, M. Siddiq, N. Shahzad, R. Ihsan, A. Abrar, and M. I. Shahzad, Renewable Sustainable Energy Rev., 119, 109549 (2020).

3. E. Irisarri, A. Ponrouch, and M. R. Palacin, J. Electrochem. Soc., 162, A2476 (2015).

4. E. Irisarri, N. Amini, S. Tennison, C. Matei Ghimbeu, J. Gorka, C. Vix-Guterl, A. Ponrouch, and M. R. Palacin, J. Electrochem. Soc., 165, A4058 (2018).

5. R. Morita, K. Gotoh, M. Fukunishi, K. Kubota, S. Komaba, N. Nishimura, T. Yumura, K. Deguchi, S. Ohki, T. Shimizu, and H. Ishida, J. Mater. Chem. A, 4, 13183 (2016).

6. H. Yamamoto, S. Muratsubaki, K. Kubota, M. Fukunishi, H. Watanabe, J. Kim, and S. Komaba, J. Mater. Chem. A, 6, 16844 (2018).

7. X. Zhou and Y. Guo, ChemElectroChem, 1, 83 (2014).

8. B. Zhang, C. M. Ghimbeu, C. Laberty, C. Vix-Guterl, and J. M. Tarascon, $A d v$. Energy Mater, 6, 1501588 (2016).

9. H. Ying and W. Q. Han, Adv. Sci., 4, 1700298 (2017).

10. H. Mou, W. Xiao, C. Miao, R. Li, and L. Yu, Front Chem., 8, 141 (2020).

11. M. Fukunishi, T. Horiba, M. Dahbi, K. Kubota, S. Yasuno, and S. Komaba, Electrochemistry, 87, 70 (2019).

12. Y. Xu, Y. Zhu, Y. Liu, and C. Wang, Adv. Energy Mater, 3, 128 (2013).

13. M. K. Datta, R. Epur, P. Saha, K. Kadakia, S. K. Park, and P. N. Kumta, J. Power Sources, 225, 316 (2013).

14. C. Kim, K. Lee, I. Kim, J. Park, G. Cho, K. Kim, J. Ahn, and H. Ahn, J. Power Sources, 317, 153 (2016).

15. F. Pan, Y. Cao, M. Xu, L. Shao, J. Sun, and A. Chen, Mater. Lett., 273, 127909 (2020).

16. S. Shimizu, H. B. Rajendra, R. Watanuki, and N. Yabuuchi, Electrochemistry, 87, 276 (2019).

17. S. Kawakami and K. Kanamura, Electrochemistry, 82, 467 (2014).

18. S. Sato, Kinzoku, Materials Science \& Technology, 80, 1017 (2010) [in Japanese].

19. T. Li, U. Gulzar, R. P. Zaccaria, C. Capiglia, S. A. Hackney, and K. E. Aifantis, J. Phys. Chem. C, 123, 15244 (2019). 